\title{
Favorable Effects of Sacubitril/Valsartan on the Peak Atrial Longitudinal Strain in Patients With Chronic Heart Failure and a History of One or More Episodes of Atrial Fibrillation: A Retrospective Cohort Study
}

\author{
Renato De Vecchis ${ }^{\mathrm{a}, \mathrm{d}}$, Andrea Paccone ${ }^{\mathrm{b}}$, Marco Di Maio ${ }^{\mathrm{c}}$
}

\begin{abstract}
Background: The peak atrial longitudinal strain (PALS) is primarily an index of the reservoir function of atrial chambers. The conceptual basis exists to hypothesize that sacubitril/valsartan improves the expandability of atrial chambers in the reservoir phase of the atrial mechanical cycle, as a consequence of its effect of prolonging the half-life of natriuretic peptides. Therefore in this retrospective study we evaluated the repercussions of the administration of sacubitril/valsartan maintained for at least 12 months on the PALS.
\end{abstract}

Methods: In our retrospective study a cohort of 40 patients treated with sacubitril/valsartan has been compared with a second cohort subjected to the conventional treatment with an angiotensin-converting enzyme (ACE) inhibitor or angiotensin receptor blocker. A general criterion to be satisfied was the presence of at least one episode of atrial fibrillation (AF) in the history of the enrolled patients. The study population was composed of New York Heart Association (NYHA) class II/III chronic heart failure (CHF) patients, due to the fact that the treating physicians of the patients whose clinical records were used as source of data, complied with the international guidelines that have so far validated sacubitril/valsartan exclusively for the CHF therapy. The aims were to verify whether the 1-year administration of sacubitril/valsartan is effective in improving the PALS, and also ascertain whether the drug is associated with a decreased risk of AF relapses over a mean retrospective observation period of 12 months.

Results: Sacubitril/valsartan cohort was proven to benefit from a significant increase in average values of PALS (median: 26.5\%; in-

Manuscript submitted December 24, 2019, accepted January 21, 2020

aMedical and Polyspecialist Centre, DSB 29 "S. Gennaro dei Poveri Hospital", via S. Gennaro dei Poveri 25, 80136 Naples, Italy

bDepartment of Cardiology, University of Bari “Aldo Moro", Bari, Italy

'Department of Cardiology, University of Campania "Luigi Vanvitelli”, 80138

Naples, Italy

${ }^{\mathrm{d} C}$ Corresponding Author: Renato De Vecchis, Medical and Polyspecialist Centre, DSB 29 "S. Gennaro dei Poveri Hospital", via S. Gennaro dei Poveri 25, 80136 Naples, Italy. Email: devecchis.erre@virgilio.it

doi: https://doi.org/10.14740/jocmr4076 terquartile range (IQR): $22 \%-30 \%$ ), opposed to the much less pronounced increase in PALS found in the conventional therapy cohort (median: 22.5\%; IQR: 18\% - 25.5\%). Additionally, the comparison made by means of one-way analysis of variance regarding the mean changes of PALS values, outlined clearly that the sacubitril/valsartan users had an increase in PALS after 1 year of therapy significantly greater $(\mathrm{P}<0.001)$ compared to the patients taking the conventional drugs. Moreover, a risk significantly higher of AF recurrences $(\mathrm{P}=$ 0.001 ) was identified in the conventional therapy group compared to the sacubitril/valsartan group during a 12-month retrospective observation period.

Conclusions: In the present retrospective cohort study a higher increase of PALS has been shown in the cohort treated with sacubitril/valsartan. Moreover, a reduced risk of AF recurrences has been shown in the sacubitril/valsartan users compared to the patients with CHF subjected to conventional treatment.

Keywords: Sacubitril/valsartan; Atrial fibrillation; Peak atrial longitudinal strain

\section{Introduction}

The left atrium modulates left ventricular filling through three components: a phase of reservoir or expansion during systole, a conduit phase during diastole, and an active contractile component, when sinus rhythm (SR) is present, during late diastole [1].

During paroxysmal atrial fibrillation (AF), atrial reservoir is impaired, booster functions is suppressed, whereas conduit function is unaltered [2], except for the fact that the temporal segments of various duration constituting the conduit phase are more numerous, given the higher heart rate usually found in paroxysmal AF compared to SR.

Increased reservoir function may play an important role in accelerating left ventricular filling by helping to maintain an enhanced atrioventricular pressure gradient during diastole and also by increasing left atrial booster function through an increase in preload. The ability to optimally redistribute left ventricular filling among reservoir, conduit and booster pump 
functions is a potentially important adaptation that may occur in the left atrium in response to changing hemodynamics [3]. An important query is whether any drugs exist able to specifically improve the function of the reservoir phase of the atrial mechanical cycle. This issue is at the moment largely unanswered, however the attempt to travel this very fascinating research pathway seems reasonable to us. The reservoir phase has been shown to be systematically impaired on the occasion of an episode of AF [4]. Moreover, after cardioversion of an episode of AF, the patients mostly prone to develop AF recurrences are just those with the most deteriorated reservoir atrial phase, identificated by means of speckle tracking echocardiography (STE) [5]. Therefore, the study of the atrial reservoir phase, corresponding to the ventricular systole, should be deemed an important step in the diagnostic and prognostic workup for both the patients with AF and those with SR at high risk of developing AF. In particular, the agents exploited in the AF therapy (beta-blockers, verapamil, amiodarone, propafenone, etc.), exert their rate-lowering effect mainly by means of a diastole prolongation. Their use has been proven to be advantageous, especially for patients still working on the ascending branch of the Frank-Starling curve. Indeed, this approach translates into an advantage as the increased preload, bradycardia-related, causes an augmented initial length of the ventricular myocardial fibers resulting in an increased stroke volume.

A very remarkable advancement might be the availability of drugs that counteract AF not just by lengthening the diastole, but also by increasing the atrial expandability occurring at the atrial reservoir stage.

We therefore verified with a retrospective cohort study, conducted on a small sample of patients with a history of AF, whether the peak atrial longitudinal strain (PALS) values were significantly influenced by an agent known for its tropism on the atrial natriuretic peptide, namely the sacubitril/valsartan. Notably, this is the first case of an observational study centered on sacubitril/valsartan used as a drug for the secondary prevention of AF. Based on a retrospective cohort study in patients with a history of AF, the aims were to verify whether the 1-year administration of sacubitril/valsartan is effective in improving the PALS and also ascertain whether the drug is associated with a decreased risk of AF relapses during a mean retrospective observation of 12 months.

\section{Materials and Methods}

The present retrospective cohort study has been derived from the findings of two centers, whose patients have been included, in the event that they comply with the following criteria: patients with SR, all with history of paroxysmal, persistent or long-lasting persistent AF, converted to SR by means of pharmacological or electrical cardioversion. All patients had to be affected by chronic heart failure (CHF), New York Heart Association (NYHA) class II or II - III. Some contraindications were provided for, as follows: recent, i.e., dating back to less than 6 months, acute myocardial infarction, well-documented systemic or pulmonary thromboembolism, absolute contraindications to the practice of therapy with an oral anticoagulant, such as Werlhof's disease or cirrhosis of the liver. Data were gathered from patients' clinical records, and for every patient the clinical pathway inferable from the consultation backwards of his clinical sheets was traced. The study population was composed of patients subject to periodical clinical, electrocardiographic and echocardiographic examinations following at least one AF episode requiring pharmacological or electrical cardioversion. No authorization was required from the Ethics Committees of the two hospitals from which the patients came. Instead, active cooperation and participation was required to the Health Departments, with the modalities of a technical support devoted to provide aid and assistance for the purposes of the consultation of the electronic files. Furthermore, the greatest attention was paid to the observance of the rules for preserving individual privacy and anonymity, which translated into the adoption of appropriate measures such as the names' concealment, as well as the obscuration of all possible sources of information that would have allowed to trace the identity of the patients constituting the study population. In order to prevent the results from being subjected to the crucial influence of unbalanced drug interferences, a great attention was paid to the nature and quantity of the drugs administered. In effect, from a repertoire of drugs as homogeneous as possible, a uniform distribution of the confounding pharmacological factors was derived. Importantly, the authors of the present retrospective cohort study did not have any decisional role in the therapies, but they merely accomplished the task of acquiring and subsequently processing the data for scientific research. The doctors who had prescribed and implemented the therapies did not participate in the statistical processing of the data. Likewise, they had no role in the writing of the present article. Furthermore, it was not consistently possible to identify a rational criterion, capable of systematically justifying the indication of the doctors to use sacubitril/ valsartan or alternatively, to prefer conventional therapy. In fact, the reason for the switch from therapy with angiotensinconverting enzyme (ACE) inhibitors or angiotensin receptor blockers (ARBs) to that with sacubitril/valsartan was usually not specified in the clinical sheets.

In each of the two cohorts the treatment had to be accurately diversified: in the cohort including the investigational treatment, that is the sacubitril/valsartan, therapy should have consist in a regular administration of sacubitril/valsartan adequately up-titrated over time (firstly $50 \mathrm{mg}$ twice daily, subsequently $100 \mathrm{mg}$ twice daily, and so on until the maximum tolerated dose of $200 \mathrm{mg}$ twice a day). Target doses to be reached within a reasonably short period in both the cohorts were the following: for beta-blockers, a target dose was established depending on the molecule employed (for carvedilol, $25 \mathrm{mg}$ twice daily, for bisoprolol $5 \mathrm{mg}$ twice daily, and for nebivolol $5 \mathrm{mg}$ once a day). In addition, for mineralocorticoid receptor antagonists (MRAs), a daily target dose of $75 \mathrm{mg}$ for spironolactone and $50 \mathrm{mg}$ for both canrenone and eplerenone was established.

Furthermore, exclusively limited to cohort subject to the conventional treatment, the dosing of ACE inhibitors or ARBs was diversified in an appropriate manner. In particular, the daily target dose was $20 \mathrm{mg}$ for enalapril, $10 \mathrm{mg}$ for ramipril, and $20 \mathrm{mg}$ for lisinopril. Likewise, as regards ARBs, the daily dos- 
es of $225 \mathrm{mg}$ for irbesartan, $100 \mathrm{mg}$ for losartan, and $160 \mathrm{mg}$ for valsartan, respectively, were deemed to be the target doses to be reached [6]. For the purposes of the homogenization, the gender and age, the comorbidities, the body mass index, the possibility of accessing routine laboratory examinations were also regarded as variables to be equally represented within the two cohorts. In this regard the propensity score matching (PSM) method was used to make the two cohorts as homogeneous and superimposable as possible. The retrospective enrollment of the cases, i.e., the patients taking sacubitril/valsartan, was based on the constitution of homogeneous groups in accordance with the PSM method [7] in order to decrease the risk of bias ensuing from the possible differences concerning the basal clinical features of the two groups. Patients on conventional therapy were paired to patients on sacubitril/valsartan with basal clinical features as similar as possible, with a $1: 1$ ratio. We applied a logistic regression model. Several variables were found to be significantly associated with the probability of belonging to one of the groups, based on a backward stepwise elimination ( $\mathrm{P}=0.05$, cut-off) methodology. The following variables were finally used to calculate the propensity score for each patient: age and care level at index date, previous anticoagulant use, antianginal drug use, insulin use, occurrence of stroke, hospitalization costs in the baseline period. Patients were matched 1:1 within gender-specific 5-years age groups, based on their propensity score with a maximum allowable difference of 0.001 .

In both cohorts the use of diuretics (loop diuretics or thiazides) was then modulated by rather lenient regulations, according to which the dosage was essentially related to the symptoms and clinical signs of congestion, i.e., rales, effort dyspnea and peripheral edema.

It is useful to remember that in the present study the population consisted of patients with $\mathrm{CHF}$ and reduced, i.e., $<40 \%$ left ventricular ejection fraction (LVEF), in accordance with the guidelines that provide for the use of sacubitril/valsartan in this category of patients. Therefore the results may not be representative of the entire clinical spectrum of the AF, but they should be referred exclusively to the secondary prevention of $\mathrm{AF}$ in CHF patients. In addition, the comparison of PALS values between patients under sacubitril/valsartan and patients on conventional therapy does not arise from a prospective randomized trial but from a retrospective observational study, per se subject to the risk of bias.

\section{Equipment}

\section{Conventional echocardiography evaluation}

All the echocardiograms were performed by the same cardiologist (RDV) with specific training and experience in cardiovascular echocardiography, by using the portable Philips Sparq ultrasound machine with a $2.5 \mathrm{mHz}$ transducer.

All measurements were performed according to the Recommendations of the American Society of Echocardiography and the European Association of Cardiovascular Imaging [8].

In the subsequent analysis the following parameters were considered: left atrium indexed volume (LAVi), LVEF and the ratio of early diastolic mitral inflow velocity to early diastolic myocardial relaxation velocity (average E/e' ratio), considered a marker of diastolic ventricular function.

\section{STE}

The regional LA myocardial strain was measured by 2-dimensional (2-D) STE [9, 10]. Gray scale images of apical 4- and 2-chamber views were obtained with frame rates of $50-80 \mathrm{~Hz}$. Recordings were processed with acoustic-tracking software (EchoPAC, GE Healthcare), allowing off-line semiautomated speckle-based strain analyses (Fig. 1). Briefly, lines were manually traced along the LA endocardium. An additional epicardial line was automatically generated by the software, creating a region of interest (ROI). After manually adjusting the ROI shape, the software divided that region into six segments and generated the longitudinal strain curve. We set the zero strain point as the point just at the time of LA contractions. The PALS during ventricular systole for each of the six LA segments and mean value for all segments were then analyzed from the apical 4- and 2- chamber views, respectively. We calculated the mean values for PALS obtained from the apical 4- and 2-chamber views and defined them as the global PALS. The measurements from the five consecutive beats recorded were averaged. PALS was calculated by averaging values observed in all LA segments (global PALS), and by separately averaging values observed in the apical 4- and 2-chamber views (4- and 2- chamber average PALS). In patients in whom some segments were excluded because of the impossibility of achieving adequate tracking, PALS was calculated by averaging values measured in the remaining segments.

\section{Statistical analysis}

Continuous data were reported as means ( \pm standard deviation (SD)) or medians (interquartile range (IQR)). KolmogorovSmirnov test was used to check for normality. Comparisons between groups were performed using the Student's $t$-test or Mann-Whitney U test where appropriate. Categorical data were reported as proportions or percentages and comparisons between groups were performed using the Chi-squared test or the Fisher's exact test where appropriate. Furthermore, oneway analysis of variance (ANOVA) test was carried out for making some comparisons. To examine the changes of parameters before and after treatments (intragroup comparison), the Wilcoxon signed-rank test was applied. Statistical tests were two- tailed, and a $\mathrm{P}$ value $<0.05$ was considered statistically significant. Analyses were conducted using Excel 2016 (version 16.0, Seattle, WA, USA) as well as MedCalc Version 18.6 (Acacialaan 22, 8400 Ostend, Belgium) and Epi-Info version 7.1.5.0 for Windows (Centers for Disease Control and Prevention, Atlanta, Georgia (USA).

The Institutional Review Board (IRB) approval is not applicable/not required, considering the study features (retrospective cohort study). This study was conducted in compli- 

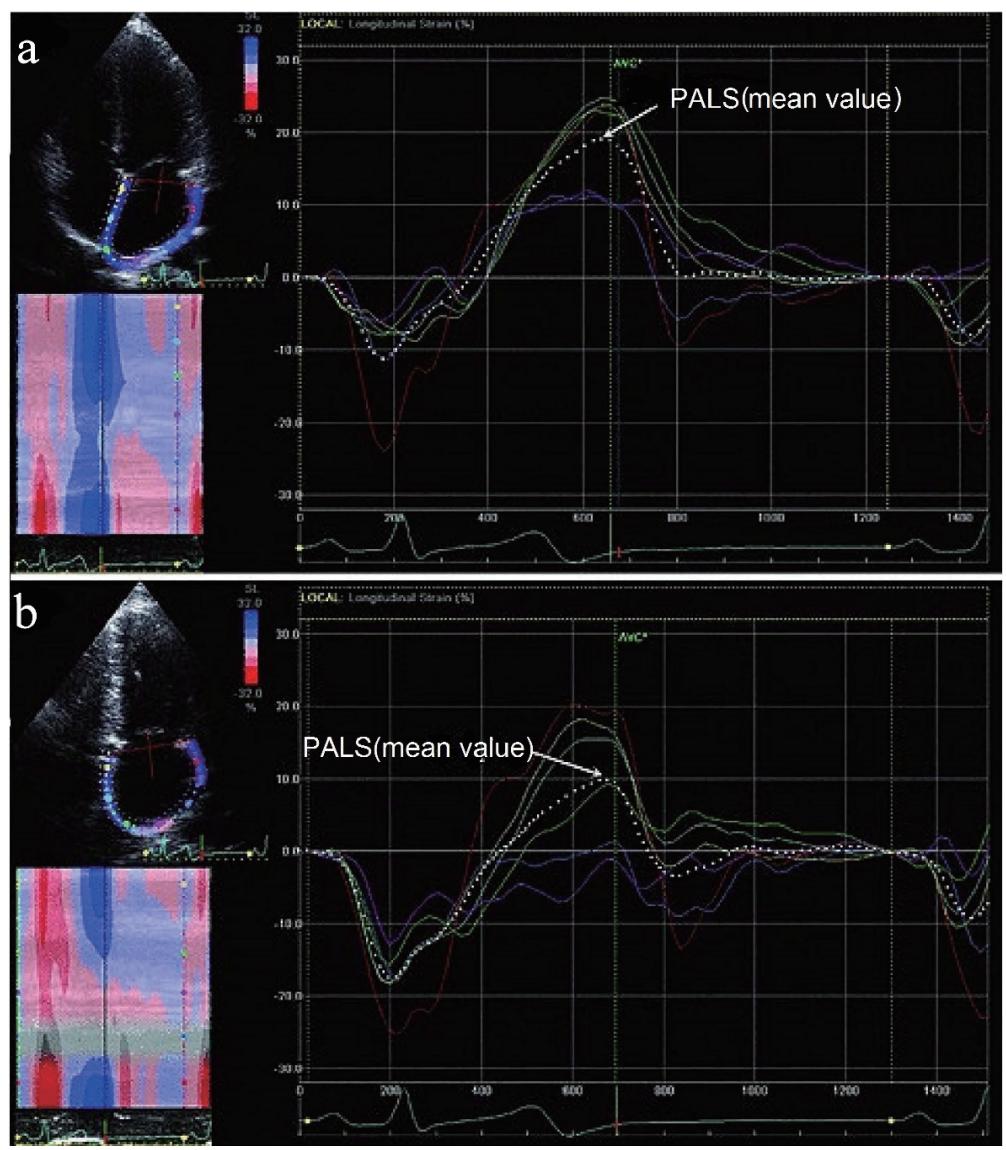

Figure 1. Examples of the longitudinal strain of the LA using 2-dimensional speckle tracking imaging. The strain (\%) of the six individually colored LA segments and mean value of all segments (dotted line) from 4-chamber and 2-chamber views are shown in (a) and (b). The PALS (mean value) refers to the mean LA peak strain value during systole. LA: left atrium; PALS: peak atrial longitudinal strain.

ance with the ethical standards of the responsible institution on human subjects as well as with the Helsinki Declaration. This study did not involve experiments on animals.

\section{Results}

On the whole, our retrospective study allowed us to identify by means of the scrupulous reading of the respective clinical records, two cohorts of patients, whose basal characteristics are outlined in Table 1. The study population encompassed 80 patients, all suffering from CHF in NYHA class II or II III. The subdivision in cohorts was exclusively based upon the nature of the medical therapy. More exactly, it was investigated whether sacubitril/valsartan was comprised in the therapy, possibly in addition to other evidence-based medications applying to the $\mathrm{CHF}$, or therapy was limited to the use of the conventional regimen providing for an ACE inhibitor or $\mathrm{ARB}$, integrating the administration of other evidence-based drugs (one at least between beta-blocker or mineralocorticoid antagonist (MRA)). Thus the discriminating determinant for the identification of a cohort was the use of sacubitril/valsartan. Moreover, the main inclusion criterion was the presence of an episode at least of AF in the clinical history. As regards the type of previous AF episode, 60 out of 80 were paroxysmal AF episodes, whereas 11 were categorized as persistent $\mathrm{AF}$, and only one met the requirements for the definition of long lasting persistent AF. The first cohort, composed of 40 patients, underwent the same examinations of atrial function, i.e., the periodical measurement of PALS, than the second cohort, also composed of 40 patients. In Table 1 the mean values of age, sex and body mass index for each of the two cohorts are also reported. An adequate emphasis was given to the measurement of PALS. The average PALS values were as follows: for the cohort encompassing the sacubitril/valsartan users ( $\mathrm{n}=40$ patients), the initial value, possibly measured immediately before the onset of the composite regimen including sacubitril/valsartan was 21\% (15.50\% - 24\%, median plus IQR). Likewise, the corresponding initial PALS value in patients subjected to conventional therapy was $21 \%$ (19\% $24 \%$, median plus IQR). Thus a substantial homogeneity of initial values for this parameter was found in the two cohorts. After 1 year, in all patients $(n=39$ patients) who had this index available in their medical records, the sacubitril/valsartan cohort was proven to benefit from a significant increase in the average values of PALS (median: $26.5 \%$; IQR: $22 \%$ - 
Table 1. Comparison of Demographics and Clinical, Laboratory, and Echocardiographic Features of Patients Examined in the Retrospective Study According to Whether or Not a CHF Patient Was Treated With Sacubitril/Valsartan

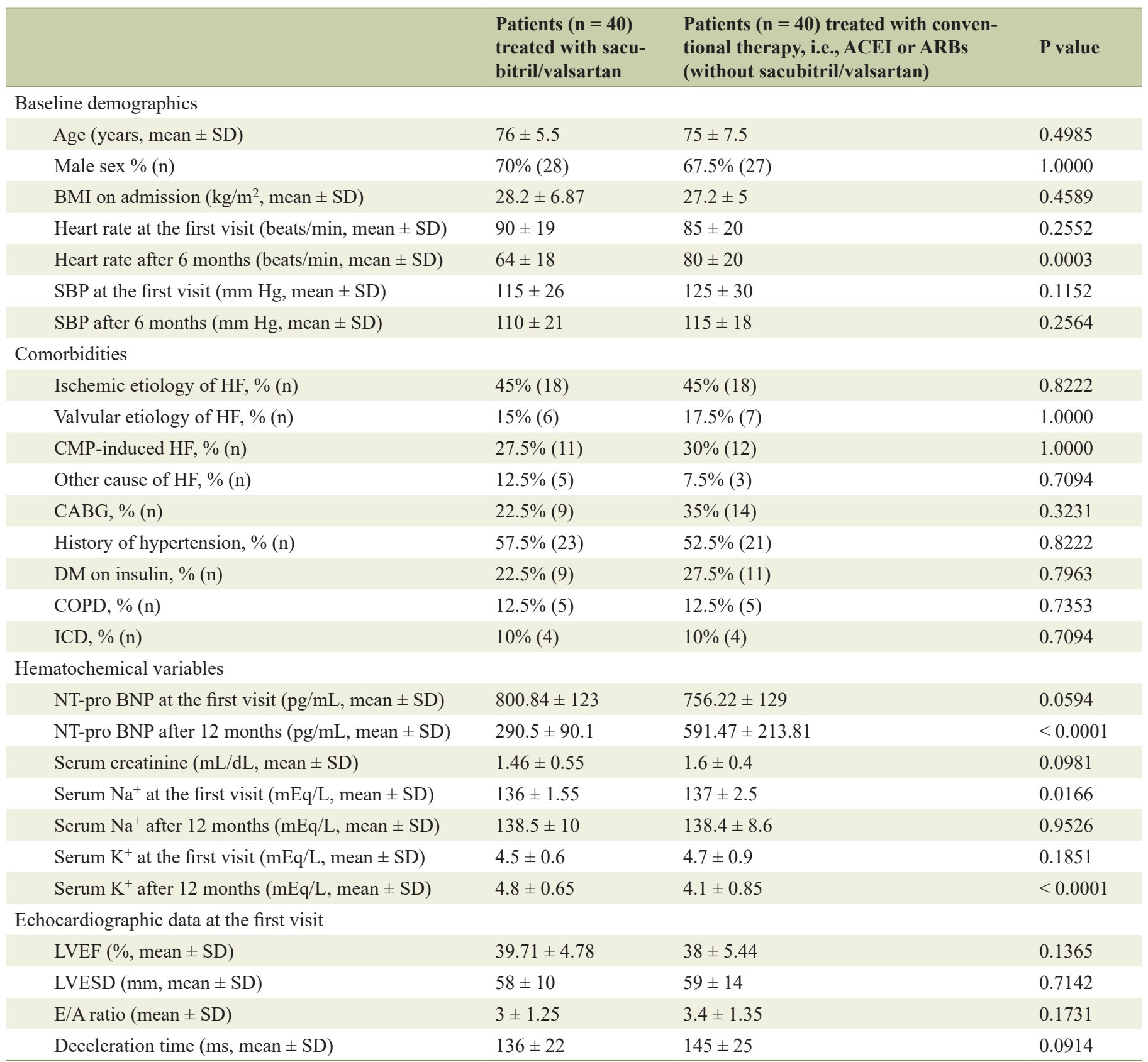

ACEI: angiotensin-converting enzyme inhibitor; ARBs: angiotensin receptor blockers; SD: standard deviation; BMI: body mass index; SBP: systolic blood pressure; HF: heart failure; CMP: cardiomyopathy; CABG: coronary artery bypass graft; DM: diabetes mellitus; COPD: chronic obstructive pulmonary disease; ICD: implantable cardioverter defibrillator; LVEF: left ventricular ejection fraction; LVESD: left ventricular end-systolic diameter; BNP: B-type natriuretic peptide.

$30 \%$ ) (Fig. 2), opposed to the much less pronounced increase in PALS found in the conventional therapy cohort (median: $22.5 \%$; IQR: $18 \%$ - 25.5\%). Additionally, the comparison made by means of one way ANOVA (Fig. 3), regarding the mean changes of PALS values, outlined clearly that the sacubitril/valsartan users had an increase in PALS after 1 year of therapy significantly greater $(\mathrm{P}<0.001)$ compared to the patients taking the conventional drugs.

As regards the objective of verifying whether the use of sacubitril/valsartan might exert a preventive effect against the AF relapses (Fig. 4), the relation between type of therapy (whether including or not sacubitril/valsartan) and risk of AF relapses was analyzed using the one-way ANOVA method, with the demonstration of a risk significantly higher $(\mathrm{P}=$ 


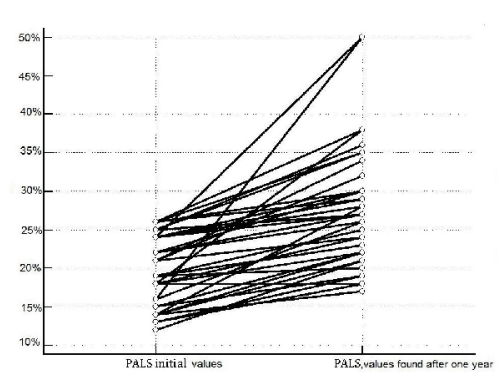

Sacubitril $f$ valsartan regimen. $P($ Wilcoxon test $)<0.0001$

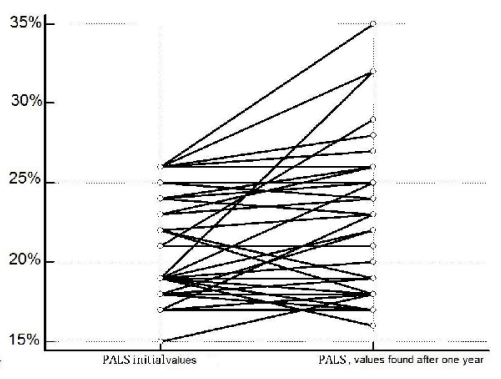

Conventional regimen. $P($ Wilcoxan test $)=0.0254$

Figure 2. During 1 year of continuous treatment respectively with sacubitril/valsartan or conventional therapy consisting of ACEI or ARBs, there are favorable effects on the mean values of PALS. In fact, the variations that occur on average after 1 year of treatment for each of the two therapeutic regimens have all a positive sign and indicate the achievement of statistical significance in both therapeutic regimens, but more marked in the sacubitril/valsartan group, whose $P$ value (Wilcoxon test) is $<0.0001$ versus a $P$ value $($ Wilcoxon test $)=0.0254$ detected in the group subject to conventional therapy. PALS: peak atrial longitudinal strain; ACEI: angiotensin-converting enzyme inhibitor; ARB: angiotensin receptor blocker.

\begin{tabular}{|c|c|c|c|}
\hline Data & \multicolumn{3}{|l|}{ change_in_PALS } \\
\hline $\begin{array}{l}\text { Factor codes } \\
\text { Sample size }\end{array}$ & \multicolumn{3}{|l|}{$\begin{array}{l}\text { therapy } \\
80\end{array}$} \\
\hline \multicolumn{4}{|l|}{ ANOVA } \\
\hline Source of variation & Sum of squares & DF & Mean square \\
\hline $\begin{array}{l}\text { Between groups } \\
\text { (influence factor) }\end{array}$ & 858.0500 & 1 & 858.0500 \\
\hline $\begin{array}{l}\text { Within groups } \\
\text { (other fluctuations) }\end{array}$ & 1981,5000 & 78 & 25,4038 \\
\hline Total & 2839.5500 & 79 & \\
\hline F-ratio & & & 33,776 \\
\hline Significance level & & & $P<0,001$ \\
\hline \multicolumn{4}{|c|}{ Student-Newman-Keuls test for all pairwise comparisons } \\
\hline Factor & $\mathrm{n}$ & Mean & $\begin{array}{l}\text { Different }(P<0,05) \\
\text { from factor } n r\end{array}$ \\
\hline (1) CONVENTIONAL & 40 & 1,1500 & (2) \\
\hline (2) SACUBV & 40 & 7,7000 & (1) \\
\hline
\end{tabular}

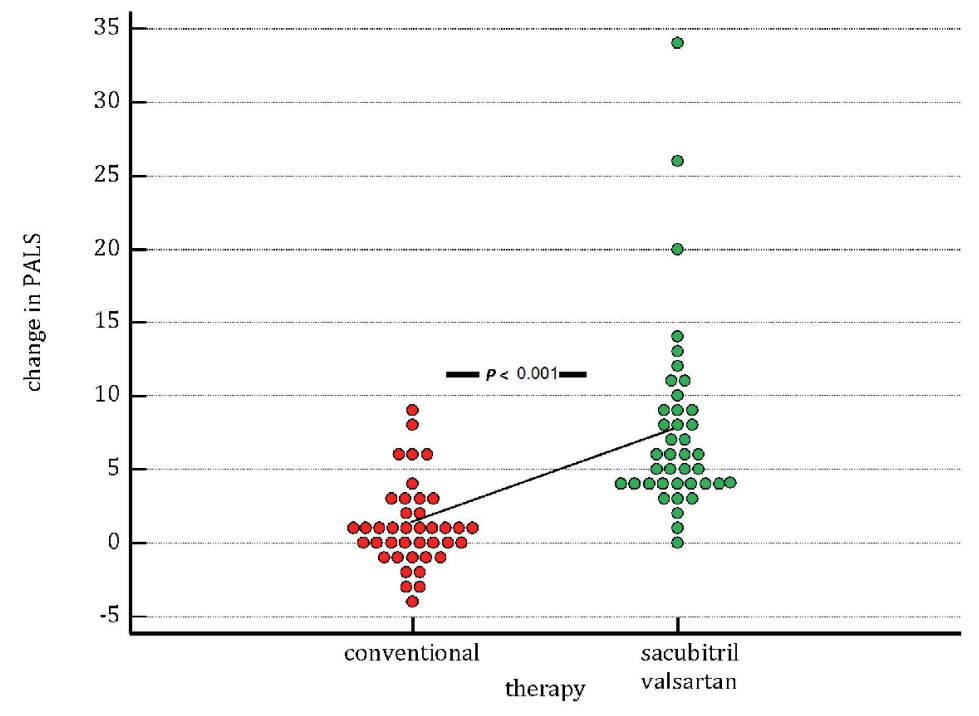

Figure 3. One of the main aims of the study was to verify whether the administration of sacubitril/valsartan (sac/v) maintained for 1 year is effective in improving the PALS in patients at risk for atrial fibrillation (AF) episodes. For this purpose it was necessary to recruit retrospectively a sufficient number of patients that had experienced at least one episode of AF successfully converted with the use of electrical or pharmacological cardioversion. According to the rules that apply to the administration of sac/v, the recruited patients had to suffer from chronic II - III NYHA class heart failure. Based on clinical considerations that were not always expressed in the clinical records, some patients were assigned to sac/v as a substitute of the traditional therapy with ACEI or ARBs, whereas others practiced the conventional therapy. The figure highlights that patients assigned to sac/v (green dots) have significantly greater increase in PALS (P (ANOVA) < 0.001) compared to patients subjected to conventional therapy (red dots). A line conjoins the mean changes in PALS of the two groups. PALS: peak atrial longitudinal strain; AF: atrial fibrillation; NYHA: New York Heart Association; ACEI: angiotensin-converting enzyme inhibitor; ARB: angiotensin receptor blocker; ANOVA: analysis of variance. 


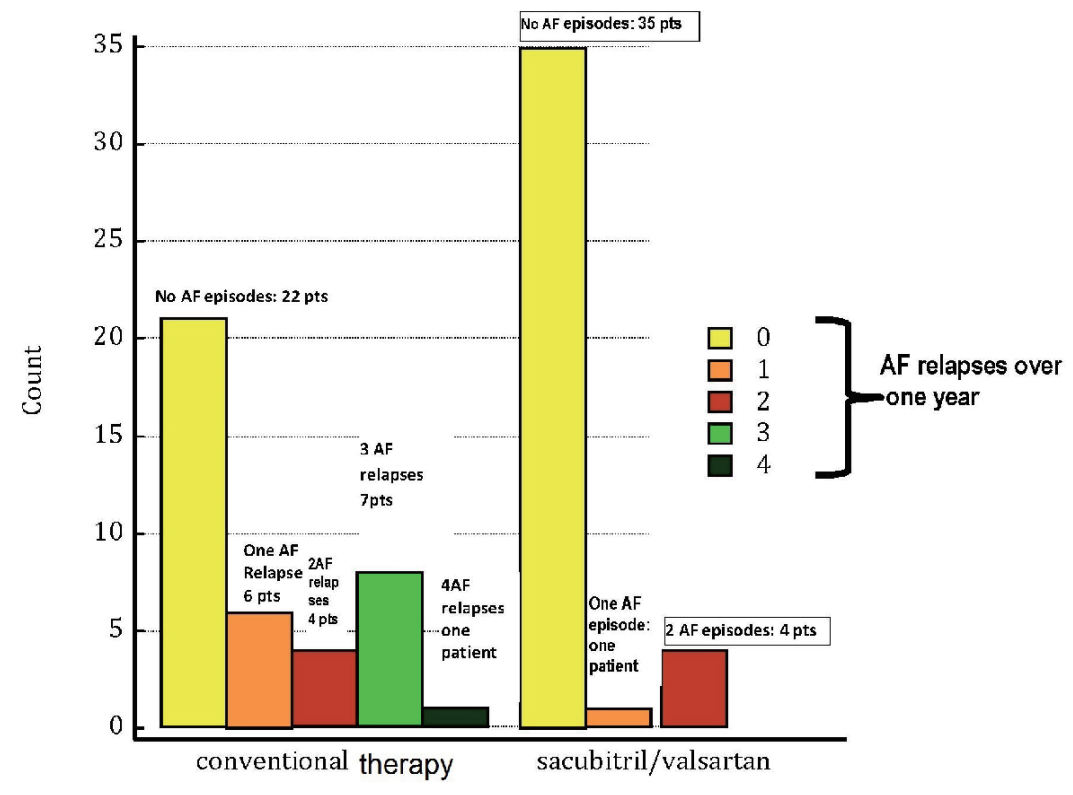

Figure 4. The atrial fibrillation (AF) relapses in patients subjected to conventional therapy are compared with those of patients who experienced therapy with sacubitril/valsartan to replace ACEI or ARBs. All of the patients were enrolled retrospectively and had to have suffered from at least one episode of AF converted to sinus rhythm with electrical or pharmacological cardioversion. The count of AF episodes recorded during 1 year of conventional therapy or sacubitril/valsartan regimen shows the above reported results, as visually represented by the colored bars (see also the Results section in the text). On the whole, a superior incidence of AF relapses is noticeable in the group of "conventional therapy", which encompasses also the cases of patients with multiple episodes; whereas sacubitril/valsartan regimen comprises only four patients whose AF burden reaches the threshold of two AF episodes during 1 year. Thus, an incisive action against the occurrence of AF in chronic heart failure patients is inferable in the cohort treated with sacubitril/valsartan. AF: atrial fibrillation; ACEI: angiotensin-converting enzyme inhibitor; ARB: angiotensin receptor blocker; pts: patients.

0.001) in the conventional therapy group.

\section{Discussion}

This retrospective cohort study has explored the issues of the responsivity of the atrial dynamics to the complex action of sacubitril/valsartan. The fact that this drug has been proven to favorably interfere with the function of atrial reservoir means really that the drug is able to advantageously modulate the phases of the cardiac mechanical cycle that are simultaneous with the reservoir stage of the atrial chambers, namely the isovolumic contraction of the ventricular myocardium, its ejective systole and the subsequent isovolumic relaxation. When a drug increases significantly the PALS, a finding that can be detected exclusively with the use of STE, this increase should be emphasized as a proof that the drug promotes the filling of the atrial chambers and thereby favors a more pronounced ejection of the left ventricle during systole. Therefore the harmonious myocardial relaxation involves not only the ventricular myocardial fibers, but also those of the atria. The result of the delayed degradation of the natriuretic peptides, due to the sacubitril/valsartan, implies that the atrial natriuretic peptide becomes able to display in full its compelling property to favor the atrial mechanics via greater atrial wall expandability.

In particular, in our study, 40 patients taking sacubitril/ valsartan, whose basal features had few dissimilarities with the control group taking conventional therapy have been compared with the latter as regards the ability to promote an increase of the PALS over time. Additionally, the two cohorts have been compared as regards the potential property of the drug to prevent the AF relapses. The results would seem support the administration of sacubitril/valsartan, because it proves to be an agent able to augment the longitudinal atrial deformation, and perhaps via this beneficial effect it is shown to be able also to reduce the frequency of AF recurrences, when compared with the conventional treatment in the course of 1-year follow-up.

Other ancillary considerations could be made. It could be said that sacubitril/valsartan is absolutely devoid of positive inotropic effect, yet it improves over time the mechanical efficiency of the heart both by favoring a greater systolic deformation of the left ventricular chamber along the longitudinal axis, and by promoting greater blood aspiration from the pulmonary veins and venous caval system toward the atria during the atrial reservoir phase. These effects are due to the balanced enhancement of the physiological effects of the two natriuretic peptides both the atrial natriuretic peptide (ANP) and the Btype natriuretic peptide (BNP) produced by the ventricular myocardium. In short, this drug amplifies and prolongs the beneficial effects of the cardioprotective hormones. Even the ameliorated blood aspiration by atrial reservoir, as well as the improved base-to-apex contraction results from the magnification of the effects of these cardioprotective hormones ensuing from the neprilysin inhibition. 


\section{Conclusions}

In the present retrospective cohort study a greater increase of PALS has been shown in the cohort treated with sacubitril/valsartan. Moreover, a reduced risk of AF recurrences has been shown in the sacubitril/valsartan users compared to the patients with CHF subjected to conventional treatment.

\section{Acknowledgments}

None to declare.

\section{Financial Disclosure}

The authors Renato De Vecchis, Andrea Paccone and Marco Di Maio declare that the present article has not benefitted from any source of funding.

\section{Conflict of Interest}

The authors Renato De Vecchis, Andrea Paccone and Marco Di Maio do not have any conflict of interest to declare concerning the present article.

\section{Informed Consent}

Not applicable.

\section{Author Contributions}

RDV and AP contributed to conceptualization; RDV contributed to data curation; RDV, AP and MDM contributed to writing and original draft preparation; RDV, AP and MDM contributed to writing, review and editing of the manuscript.

\section{Data Availability}

The data supporting the findings of this study are available from the corresponding author upon reasonable request.

\section{References}

1. Cameli M, Mandoli GE, Loiacono F, Sparla S, Iardino E, Mondillo S. Left atrial strain: A useful index in atrial fibrillation. Int J Cardiol. 2016;220:208-213.

2. Stefanadis C, Dernellis J, Toutouzas P. A clinical appraisal of left atrial function. Eur Heart J. 2001;22(1):22-36.

3. Yoon YE, Kim HJ, Kim SA, Kim SH, Park JH, Park KH, Choi S, et al. Left atrial mechanical function and stiffness in patients with paroxysmal atrial fibrillation. J Cardiovasc Ultrasound. 2012;20(3):140-145.

4. Donal E, Galli E, L'Official G. The left atrium: A reservoir and a witness for risk of symptoms and cardiovascular complications. Eur J Prev Cardiol. 2019;26(10):10151017.

5. Ancona R, Comenale Pinto S, Caso P, D'Andrea A, Di Salvo G, Arenga F, Coppola MG, et al. Left atrium by echocardiography in clinical practice: from conventional methods to new echocardiographic techniques. ScientificWorldJournal. 2014;2014:451042.

6. De Vecchis R, Ariano C, Di Biase G, Noutsias M. Sacubitril/valsartan for heart failure with reduced left ventricular ejection fraction : A retrospective cohort study. Herz. 2019;44(5):425-432.

7. Grotta A, Bellocco R. A review of propensity score: principles, methods and application in Stata. Italian Stata Users Group Meeting - Milano. 13 November 2014. p. 2-64.

8. Lang RM, Badano LP, Mor-Avi V, Afilalo J, Armstrong A, Ernande L, Flachskampf FA, et al. Recommendations for cardiac chamber quantification by echocardiography in adults: an update from the American Society of Echocardiography and the European Association of Cardiovascular Imaging. Eur Heart J Cardiovasc Imaging. 2015;16(3):233-270.

9. D'Andrea A, Caso P, Romano S, Scarafile R, Riegler L, Salerno G, Limongelli G, et al. Different effects of cardiac resynchronization therapy on left atrial function in patients with either idiopathic or ischaemic dilated cardiomyopathy: a two-dimensional speckle strain study. Eur Heart J. 2007;28(22):2738-2748.

10. Hwang HJ, Choi EY, Rhee SJ, Joung B, Lee BH, Lee SH, Kim J, et al. Left atrial strain as predictor of successful outcomes in catheter ablation for atrial fibrillation: a two-dimensional myocardial imaging study. J Interv Card Electrophysiol. 2009;26(2):127-132. 